\title{
AVALIAÇÃO DA GERMINAÇÃo SEMENTES DE JABUTICABA (Myrciaria cauliflora) PÓS-ARMAZENAMENTO, EM DIFERENTES CONDIÇÕES AMBIENTAIS
}

\author{
Daniel Soares Ferreira ${ }^{1}$ \\ Laura Vaillant Ribeiro ${ }^{2}$ \\ Wilian Rodrigues Ribeiro ${ }^{3}$ \\ Ualace de Oliveira do Reis ${ }^{4}$ \\ Paula Aparecida Muniz de Lima ${ }^{5}$ \\ André Alves Pinheiro ${ }^{6}$ \\ Vinicius Agnolette Capelini ${ }^{7}$ \\ José Carlos Lopes ${ }^{8}$
}

\begin{abstract}
Resumo: A semente de jabuticaba por possuir um gral de recalcitrância exige cuidados especiais no póscolheita. Objetivou-se neste experimento analisar duas formas de armazenagem de sementes de jabuticaba e determinar o método mais eficiente que preserve as características da semente. O presente trabalho foi desenvolvido no laboratório de análise de sementes, da Universidade Federal do Espirito Santos-UFES, localizado no município de Alegre-ES. O experimento foi montado em um delineamento experimental inteiramente casualizado com 2 tratamentos (acondicionamento no fruto e geladeira) e 4 repetições, possuindo 25 sementes por repetição. Após o período armazenado estas foram expostas à condições ideais para seu desenvolvimento onde avaliou-se as variáveis de germinação, comprimento de raiz, comprimento de plântulas, massa fresca e seca. Onde se identificou os melhores resultados em sementes armazenados em geladeira.

Palavras-chave: Recalcitrância; Armazenamento; Semente; Jabuticaba; Germinação das sementes.
\end{abstract}

\footnotetext{
1 Centro de Ciências Agrárias/Departamento de Produção Vegetal/Universidade Federal do Espirito Santo, Brasil. E-mail: danielufes@live.com.

2 Centro de Ciências Agrárias/Departamento de Produção Vegetal/Universidade Federal do Espirito Santo, Brasil. E-mail: laura.vaillant@hotmail.com.

3 Centro de Ciências Agrárias/Departamento de Produção Vegetal/Universidade Federal do Espirito Santo, Brasil. E-mail: wilianrodrigues@msn.com.

${ }^{4}$ Centro de Ciências Agrárias/Departamento de Produção Vegetal/Universidade Federal do Espirito Santo, Brasil. E-mail: ualaceoliveira@outlook.com.

5 Centro de Ciências Agrárias/Departamento de Produção Vegetal/Universidade Federal do Espirito Santo, Brasil. E-mail: alup-lima@hotmail.com.

6 Centro de Ciências Agrárias/Departamento de Produção Vegetal/Universidade Federal do Espirito Santo, Brasil. E-mail: aalvespinheiro7@gmail.com.

7 Centro de Ciências Agrárias/Departamento de Produção Vegetal/Universidade Federal do Espirito Santo, Brasil. E-mail: vinicius91@hotmail.com.

8 Centro de Ciências Agrárias/Departamento de Produção Vegetal/Universidade Federal do Espirito Santo, Brasil. E-mail: Jcufes@bol.com.br.
} 